\title{
A Wearable Cortisol Aptasensor for Simple and Rapid Real- time Monitoring
}

Jai Eun An ${ }^{\mathrm{a}, *}$, Kyung Ho Kim ${ }^{\mathrm{a}}{ }^{*}$, Seon Joo Park ${ }^{\mathrm{a}}$, Sung Eun Seo ${ }^{\mathrm{a}}$, Jinyeong Kim ${ }^{\mathrm{a}}$, Siyoung $\mathrm{Ha}^{\mathrm{a}}$, Joonwon Bae ${ }^{\text {b,* }}$, Oh Seok Kwon ${ }^{\text {a,c,** }}$

${ }^{\text {a }}$ Infectious Disease Research Center, Korea Research Institute of Bioscience and Biotechnology (KRIBB), Daejeon 34141, Republic of Korea

${ }^{\mathrm{b}}$ Department of Applied Chemistry, Dongduk Women's University, Seoul, 02748, Republic of Korea

${ }^{\mathrm{c}}$ Nanobiotechnology and Bioinformatics (Major), University of Science \& Technology (UST), 125 Gwahak-ro, Yuseong-gu, Daejeon, 34141, Republic of Korea

Corresponding author:

Joonwon Bae - Department of Applied Chemistry, Dongduk Women's University, Seoul, 02748, Republic of Korea,

Email: redsox7@dongduk.ac.kr

Oh Seok Kwon - Infectious Disease Research Center, Korea Research Institute of Bioscience and Biotechnology (KRIBB), Daejeon 34141, Republic of Korea, Nanobiotechnology and Bioinformatics (Major), University of Science \& Technology (UST), 125

Gwahak-ro, Yuseong-gu, Daejeon, 34141, Republic of Korea

Email: oskwon79@kribb.re.kr 
Table S1. The Performance Comparison Data of Various Biosensors for Cortisol Detection

\begin{tabular}{|c|c|c|c|c|}
\hline Category & Bioprobe & LOD & Wearable Application & Reference \\
\hline FET & Antibody & $270 \mathrm{pM}$ & N/A & 2 \\
\hline FET & Antibody & $28 \mathrm{pM}$ & N/A & 3 \\
\hline LFA & Aptamer & $28 \mathrm{nM}$ & Wearable patch & 4 \\
\hline FET & Aptamer & $200 \mathrm{pM}$ & N/A & 5 \\
\hline CV & Antibody & $760 \mathrm{pM}$ & N/A & 6 \\
\hline ELISA & Antibody & $41 \mathrm{nM}$ & N/A & Swappable \\
\hline CV & Antibody & $280 \mathrm{pM}$ & $10 \mathrm{pM}$ & Wearable, \\
\hline
\end{tabular}

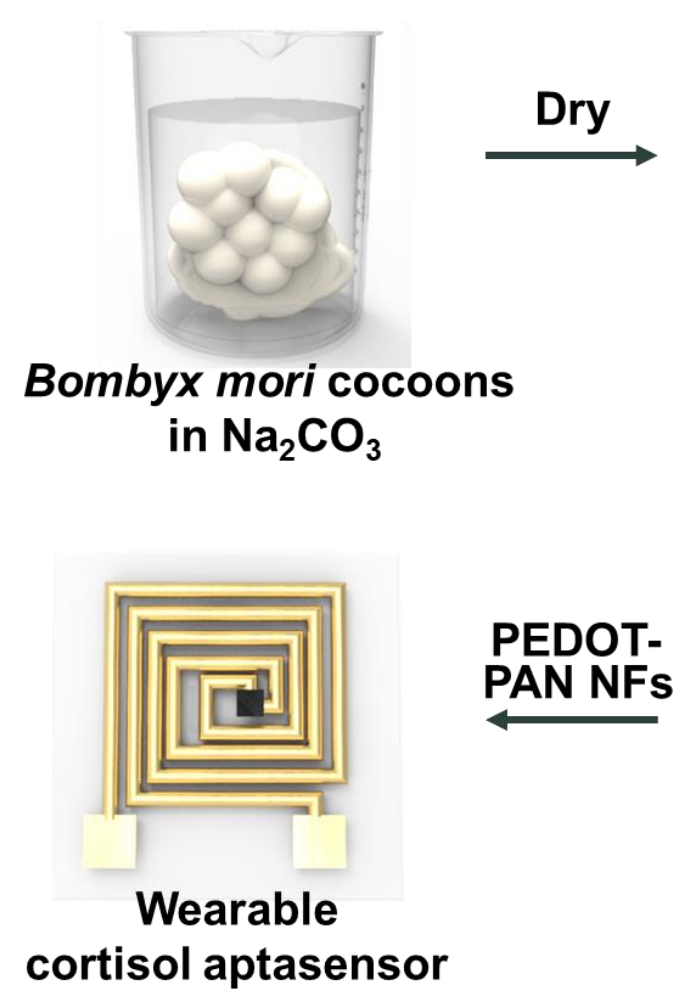

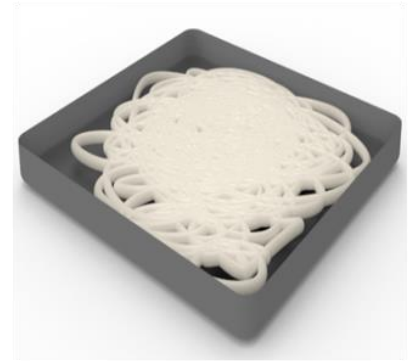

Squeezed excess

\section{Dissolution}

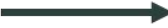

\section{Fabrication (dry overnight)}

Figure S1. Fabrication process of the silk substrate-based cortisol aptasensor. The schematic diagram showed a typical procedure to obtain a silk film, which was used as a substrate for a designed sensor system. Silk emitted by the silkworm consists of fibroin and sericin, where fibroin is the structural center of the silk surrounded by sticky sericin. To obtain a transparent and uniform thin film, separation of fibroin from the precursor is important. Fibroin is made up of the amino acids GlySer-Gly-Ala-Gly-Ala and forms beta-pleated sheets. Hydrogen bonds form between chains, and the high proportion (50\%) of glycine (Gly) allows tight packing. The addition of alanine (Ala) and serine (Ser) makes the fibers strong and resistant to breaking owing to the numerous hydrogen bonds introduced. 


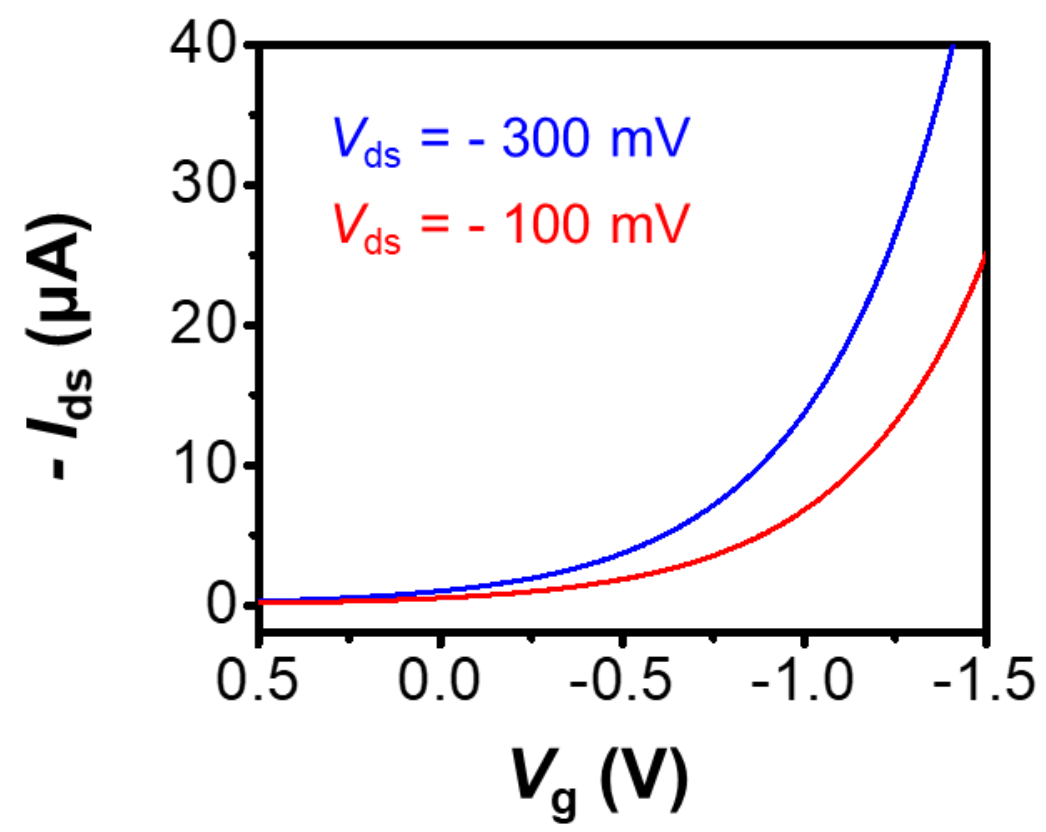

Figure S2. Transfer curve of the PEDOT-PAN NFs cortisol aptasensor using different source-drain voltages ( $\left.V_{\mathrm{ds}}\right)$.
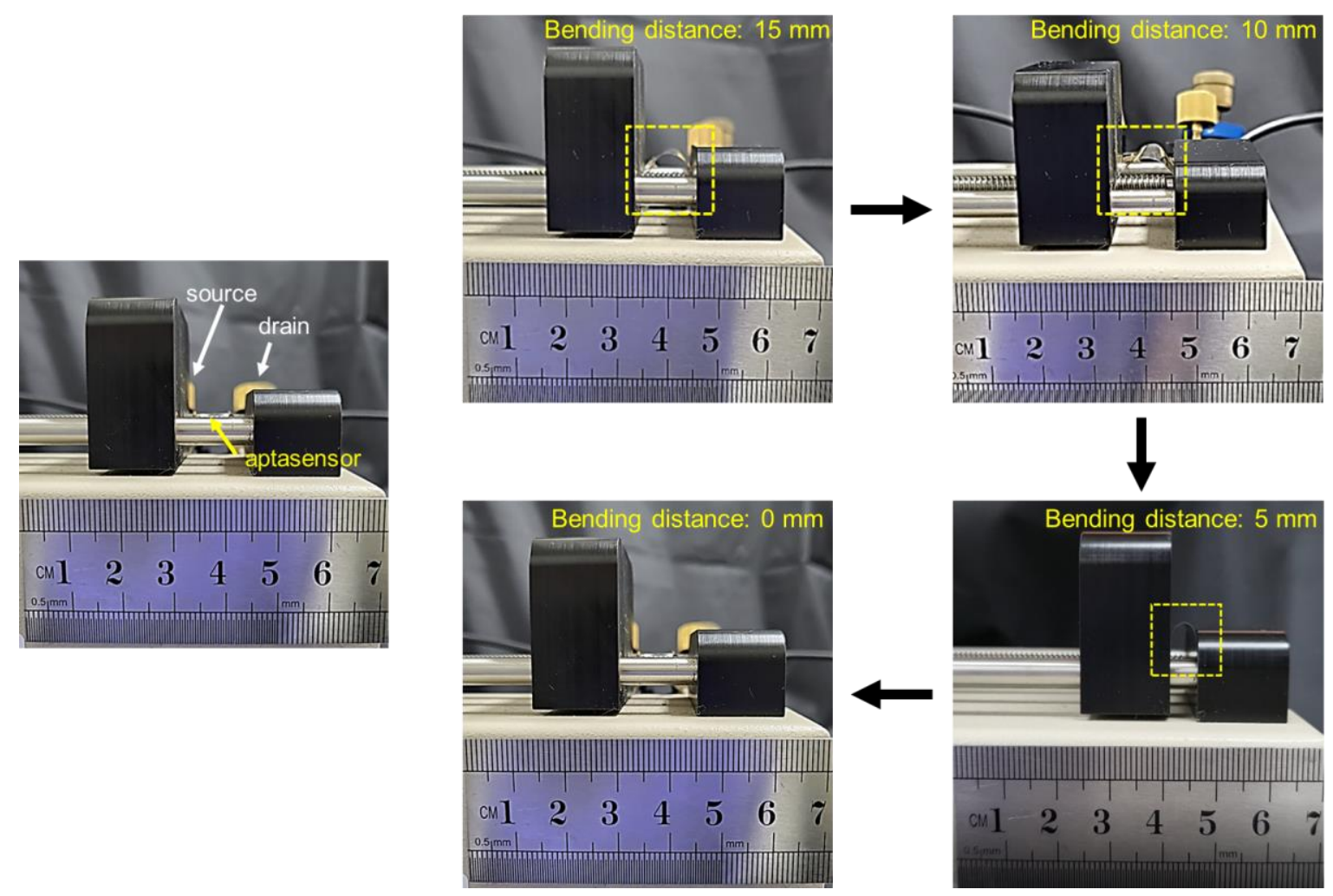

Figure S3. Enlarged actual images of the wearable cortisol aptasensor bending test. 


\section{REFERENCES}

(1) Kim, K. H.; Lee, S. H.; Seo, S. E.; Bae, J.; Park, S. J.; Kwon, O. S. Ultrasensitive Stress Biomarker Detection Using Polypyrrole Nanotube Coupled to a Field-Effect Transistor. Micromachines 2020, 11 (4), 439.

(2) Jang, H.-J.; Lee, T.; Song, J.; Russell, L.; Li, H.; Dailey, J.; Searson, P. C.; Katz, H. E. Electronic Cortisol Detection Using an Antibody-Embedded Polymer Coupled to a Field-Effect Transistor. ACS Appl. Mater. Interfaces 2018, 10 (19), 16233-16237.

(3) Dalirirad, S.; Steckl, A. J. Aptamer-Based Lateral Flow Assay for Point of Care Cortisol Detection in Sweat. Sensors Actuators B Chem. 2019, 283, 79-86.

(4) Sheibani, S.; Capua, L.; Kamaei, S.; Akbari, S. S. A.; Zhang, J.; Guerin, H.; Ionescu, A. M. Extended Gate FieldEffect-Transistor for Sensing Cortisol Stress Hormone. Commun. Mater. 2021, 2 (1), 10.

(5) Sun, K.; Ramgir, N.; Bhansali, S. An Immunoelectrochemical Sensor for Salivary Cortisol Measurement. Sensors Actuators, B Chem. 2008, 133 (2), 533-537.

(6) Kaushik, A.; Yndart, A.; Jayant, R. D.; Sagar, V.; Atluri, V.; Bhansali, S.; Nair, M. Electrochemical Sensing Method for Point-of-Care Cortisol Detection in Human Immunodeficiency Virus-Infected Patients. Int. J. Nanomedicine $\mathbf{2 0 1 5}, 10,677-685$. 